\title{
Electronic Properties of Boron and Nitrogen Doped Graphene
}

\author{
Sonai Seenithurai ${ }^{\mathrm{a}}$, Ramalingam Kodi Pandyan ${ }^{\mathrm{b}}$, \\ Shanmugam Vinodh $\operatorname{Kumar}^{\mathrm{c}}$ and Manickam Mahendran ${ }^{\mathrm{d} *}$ \\ Smart Materials Lab, Department of Physics \\ Thiagarajar College of Engineering, Madurai - 625 015, India \\ âseenithurai@tce.edu, ${ }^{b}$ rkp@tce.edu, crvinothtc@tce.edu, ${ }^{d^{*}}$ manickam-mahendran@tce.edu \\ (Corresponding Author)
}

Keywords: Graphene, B-doping, N-doping, Electronic Properties, DFT, $\mathrm{H}_{2}$ Adsorption, Charge Analysis.

\begin{abstract}
Graphene is the thinnest 2-D material which can be regarded as a single layer of graphite. The unique electrical, mechanical and optical properties of graphene can be used in many technological applications. 2-D nanomaterials with semiconducting properties are of great interest since they can be applied in electronics industry. Pure graphene is a zerogap semiconductor or semimetal, since the electron states just cross the Fermi energy. However, the electronic properties of graphene can be tuned by doping boron or nitrogen atoms. Understanding the electronic properties in terms of density of states and band structure of doped graphene is of great relevance today. In our work, we have analyzed the electronic properties of boron and nitrogen doped graphene using Density Functional Theory (DFT). The stability and charge analysis of doped structures have been studied. The Local Density Approximation (LDA) calculations have been used to find the total energies of the structures. In addition to the electronics industry, doped graphene also has great potential to adsorb gas molecules. Therefore, we have analyzed the $\mathrm{H}_{2}$ molecule adsorption in pure, B-doped and N-doped graphene.
\end{abstract}




\section{Introduction}

Graphene is the thinnest material which can be considered as single layer of graphite. Graphene is the base material for some of the carbon based nanostructures such as carbon nanotubes (CNTs), bucky ball, and nanocones. CNTs are the graphene sheets rolled in to a seamless cylinder. The carbon atoms in these nanostructures are bonded by $\mathrm{sp}^{2}$ hybridization. The carbon based nanomaterials have attracted the scientific community since their unique structural, electronic, magnetic, mechanical, optical and chemical properties [1]. Carbon nanotubes (CNTs) and graphene are proposed to have potential applications in electronics industry, hydrogen storage, biotechnology etc. Even though CNTs and graphene have remarkable properties, they are functionalized or doped to enhance or tune their properties. Doping is an efficient way to enhance the unique properties of carbon nanostructures and use them in potential applications [2-9].

As far as substitutional doping is considered, boron and nitrogen doping in CNTs and graphene is studied. The electronic properties and the hydrogen adsorption on these structures are reported in literatures [8-20]. These studies mainly focus on the local changes in the geometry, electron distribution and the doped structure's $\mathrm{H}_{2}$ adsorption behaviour. We have computed the density of states and band structure of boron and nitrogen doped graphene, using DFT. The boron concentration has been increased from 1 to 9 and the changes in the electronic structures have been analyzed. The suitability of these doped structures based on the adsorption distance and binding energy has been studied. The graphene was optimized and the density of states and band structures were calculated using DFT. Even though DFT underestimates the band gap of semi-conducting materials, this study can give some insight on the understanding of graphene based semiconductors. The HOMO-LUMO analysis have been carried our on doped structues. In addition to the structural and electronic state changes, hydrogen adsorption has also been studied in boron and nitrogen doped graphene supercell. 


\section{Methodological Approach and Computational Details}

The calculations have been done using $\mathrm{DMol}^{3}$ package as implemented in the Materials Studio [21]. The Density Functional Theory (DFT) based study was performed using Local Density Approximation (LDA) with Perdew-Wang Functional (PWC) [22]. Geometry optimization and total energy calculations were performed using spin unrestricted, periodic boundary conditions. The structures were optimized so that force on each atom is less than $0.004 \mathrm{Ha} / \AA \AA$. The convergence threshold for maximum energy change was set to $2 \times 10^{-5} \mathrm{Ha}$. An orbital cut off quality of $0.1 \mathrm{eV} /$ atom was used which corresponds to a real space cut off of $6.5 \AA$. The electronic wave functions were expanded in a double numerical polarization (DNP) basis set. All electrons were included for the calculation. The SCF tolerance was set to $10^{-5}$ and a smearing value of 0.005 Ha was used for the calculations. The binding energy of $\mathrm{H}_{2}$ adsorption was calculated using the formula-

$$
\text { B. } E_{H_{2}}=E_{\text {TOT }}\left(\text { host }-H_{2}\right)-E_{T O T}(\text { host })-E_{T O T}\left(H_{2}\right)
$$

where $\mathrm{E}_{\mathrm{TOT}}$ (host- $\mathrm{H}_{2}$ ), $\mathrm{E}_{\text {TOт }}$ (host), $\mathrm{E}_{\mathrm{TOT}}\left(\mathrm{H}_{2}\right)$ are the total energies of optimized structures of $\mathrm{H}_{2}$ adsorbed host materials, pure host materials and pure $\mathrm{H}_{2}$ molecule respectively.

\section{Results and Discussion}

Geometry and Electronic Structure of B-doped Graphene. The periodic structure of graphene was obtained from graphite. Supercell with 2 units along a and b axis was considered for the study. The cell parameters are $4.92 \AA$ along a and $\mathrm{b}$ axes. The c-axis was extended to $12 \AA$ in order to avoid interference of other sheets. The C-C bond lengths in the optimized cell are $1.42 \AA$ which is consistent with earlier literature. The $\mathrm{C}-\mathrm{C}-\mathrm{C}$ angles were measured to be $120^{\circ}$. Initially, one of the carbon atoms in the graphene was replaced by a boron atom. The optimized geometry of the Bdoped graphene shows that the distance between the doped boron atom and the nearest neigbour increases to $1.463 \AA$. A slight increase and decreae in the C-C-C angles are aso observed. However, no change is observed in C-B-C angle. The dopant concentration was increased by increasing the 
number of dopants per supercell and the structural changes are observed. When 2 boron atoms are doped, the B-C bond distance changes to a value of $1.478 \AA$. Then 4 and 9 boron atoms were doped and finally all atoms are replaced to get a boron sheet. The optimized structures of pure, 1,2,4 and 9 boron atoms doped graphene, and boron sheet are given in Fig. 1. The total energy of the graphene decreases when the boron dopant concentration increases. Notable changes in the Fermi energy and binding energy of the structures have been observed on increasing the number of boron atoms. However, no substantial changes have been observed when the number of dopant is 4 and 9 boron atoms.
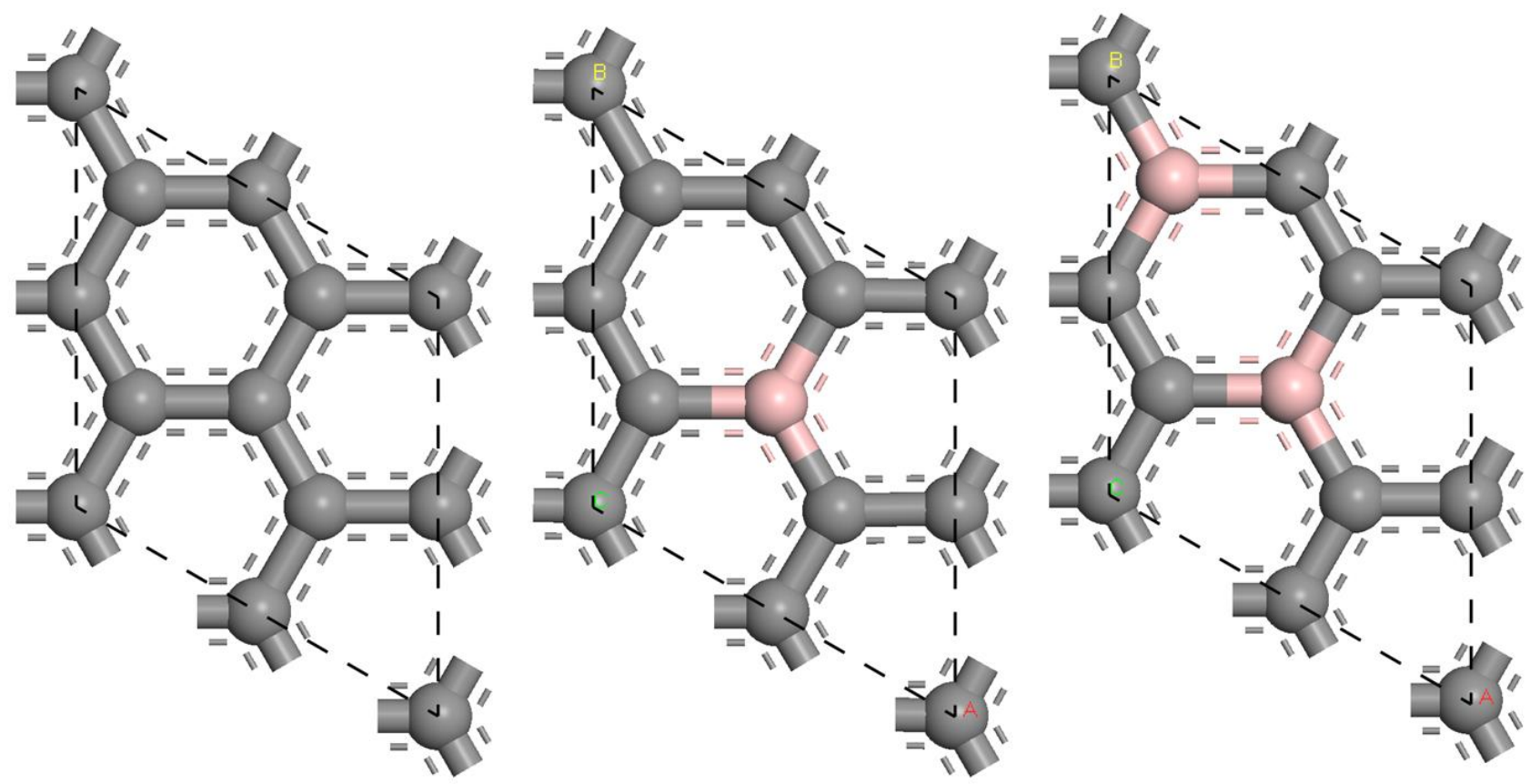


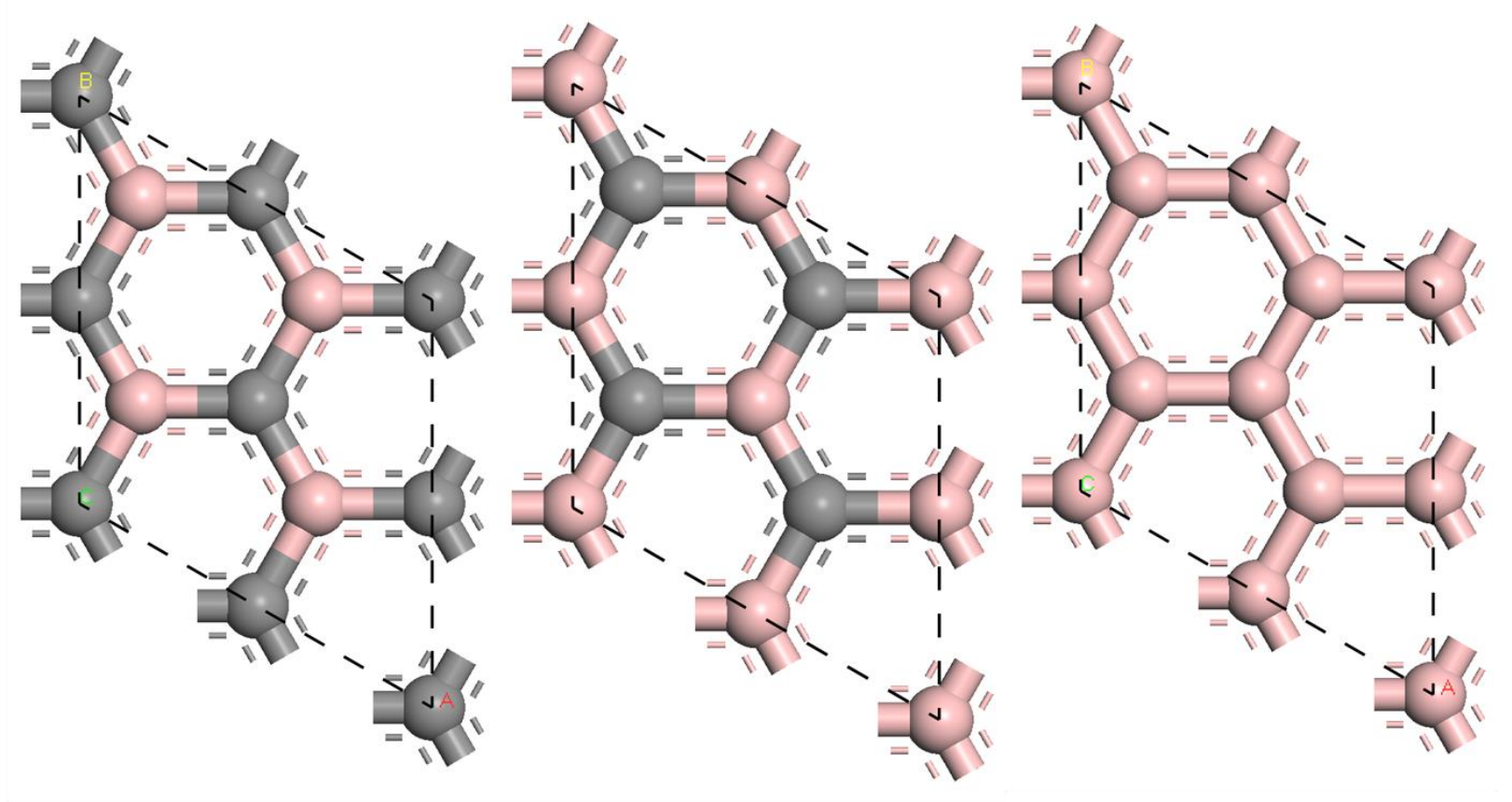

Fig. 1. The optimized structures of pure graphene, graphene doped with 1, 2, 4, 9 boron atoms and boron sheet (clockwise from top). Grey and brown coloured balls represent carbon and boron atoms respectively. The cell was constructed from the graphite. The cell dimensions are $4.92 \AA$ along a and b axis.

The density of states and band structure are computed using DFT. It is reported in many literature that DFT does not give correct band gap of semiconductors. However, DFT can give qualitative understanding on the electronic structure. The total energy, Fermi energy and binding energies of the pure and B-doped graphene are listed in Table 1. The density of states and band structures are given in Fig. 2 (a-d) and Fig. 3 (a,b) respectively. The Fermi energy of pure graphene is -0.1690 Ha. 
Table 1. Total energy, Fermi energy and binding energies of optimized structures of B-doped graphene.

\begin{tabular}{|c|c|c|c|}
\hline Structures & Total Energy [Ha] & $\begin{array}{c}\text { Fermi Energy } \\
{[\mathbf{H a}]}\end{array}$ & $\begin{array}{c}\text { Binding Energy } \\
{[\mathbf{H a}]}\end{array}$ \\
\hline Graphene (G) & 302.3343 & 0.1690 & 2.6131 \\
\hline G-1B & 289.1037 & 0.2255 & 2.4959 \\
\hline G-2B & 275.8776 & 0.2009 & 2.3890 \\
\hline G-4B & 249.3144 & 0.2119 & 2.0642 \\
\hline G-9B & 249.3144 & 0.2119 & 2.0642 \\
\hline
\end{tabular}
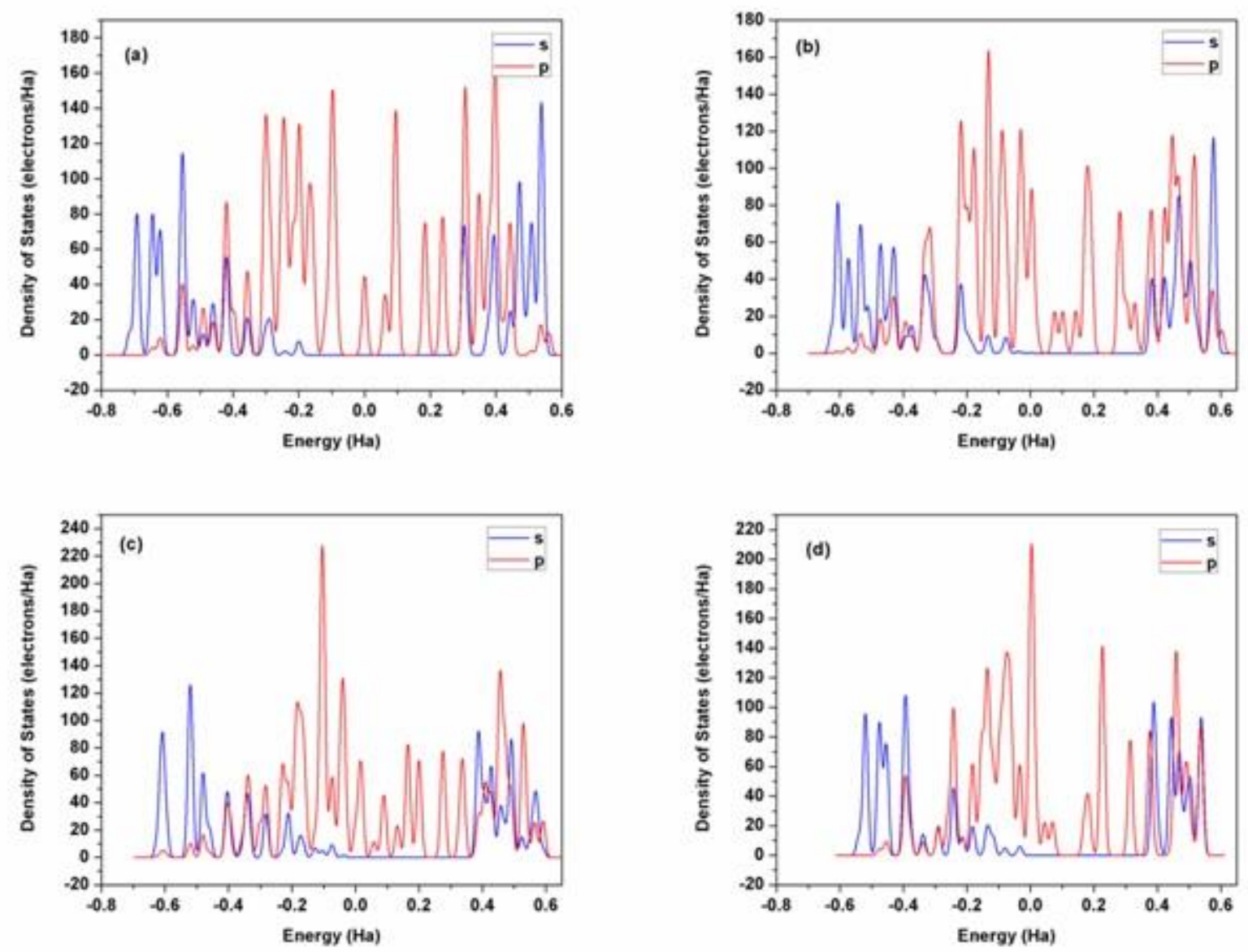

Fig. 2. Partial density of states (PDOS) of (a) pure, (b) 1B, (c) $2 B$ and (d) 4B doped graphene. The changes are observed around the Fermi energy (-0.1690 Ha) on doping with boron atoms. Blue line represents ' $s$ ' states and red line represents ' $p$ ' states. 

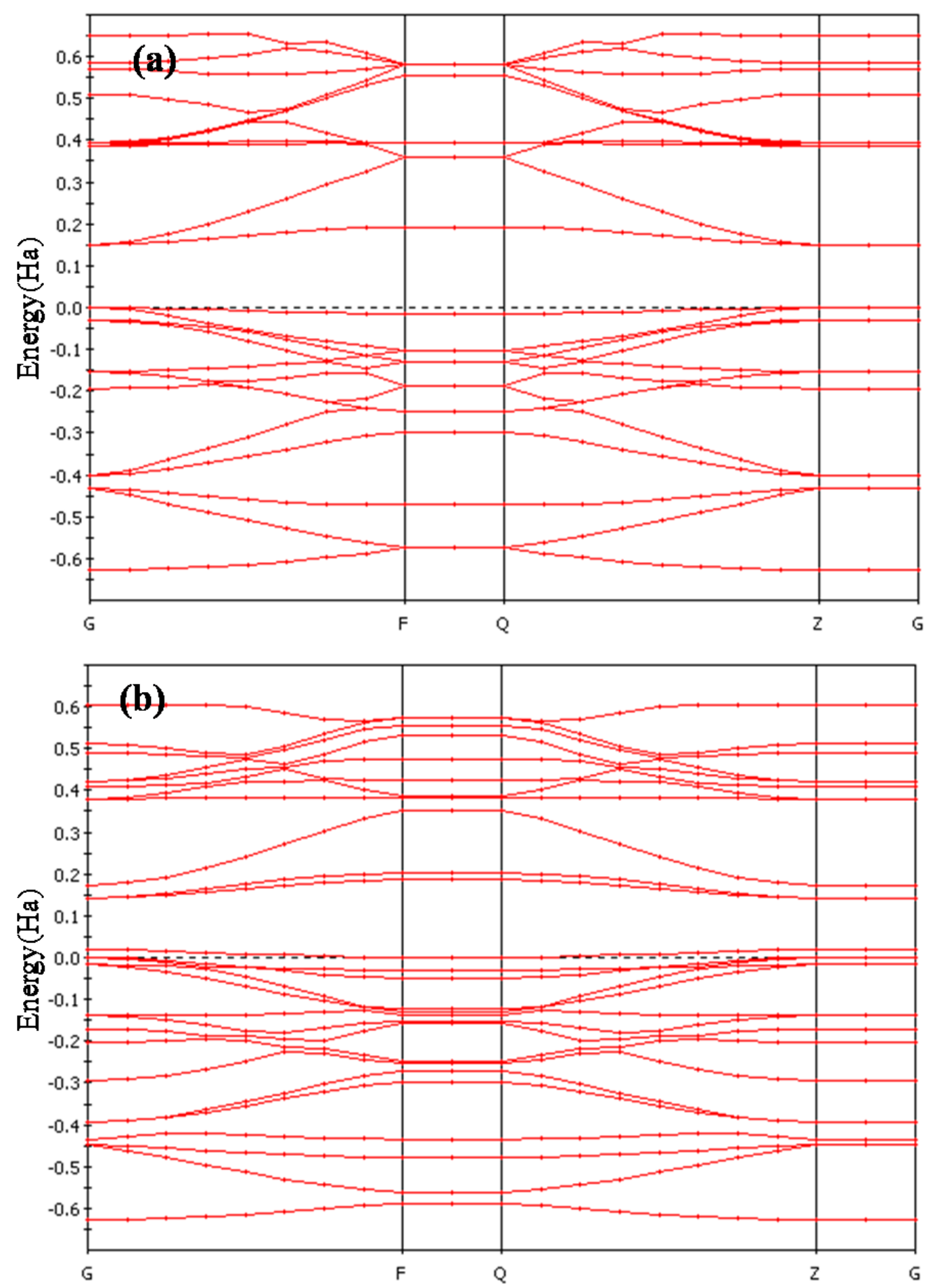

Fig. 3. Band structure of (a) pure and (b) B-doped graphene.

The density of states drastically changes on doping with boron atoms. The states near the Fermi energy change on doping boron atom. Maximum states are observed around -0.1 and $0.4 \mathrm{Ha}$ which correspond to ' $\mathrm{p}$ ' orbital. ' $\mathrm{s}$ ' and ' $\mathrm{p}$ ' orbitals completely overlap in the density of states. No states are observed around 0.15 and $0.25 \mathrm{Ha}$ for pure and B-doped graphene. For the addition of fourth 
boron atom, the maximum state is shifted towards the conduction band. Some states are absent in the four boron atoms doped graphene also. The band structure corresponding to pure graphene and Bdoped graphene is displayed in Fig. 3. Some additional bands are introduced on doping with boron atoms which is clearly understood in the figure.

Geometry and Electronic Structure of N-doped Graphene. The optimized structures of N-doped graphene are shown in Figure 4. The lattice parameters are similar to the B-doped graphene. The C$\mathrm{N}-\mathrm{C}$ bond lengths corresponding to three nearest neigbours of $\mathrm{N}$ atoms is $1.423 \AA$. A slight decrease in $\mathrm{C}-\mathrm{C}$ bond lengths is observed when one $\mathrm{N}$ atoms is doped in graphene. When two $\mathrm{N}$ atoms are doped the same change in the bond lengths are observed. The changes in the total energy, Fermi energy and binding energies corresponding to $\mathrm{N}$-doped graphene are listed in Table 2.

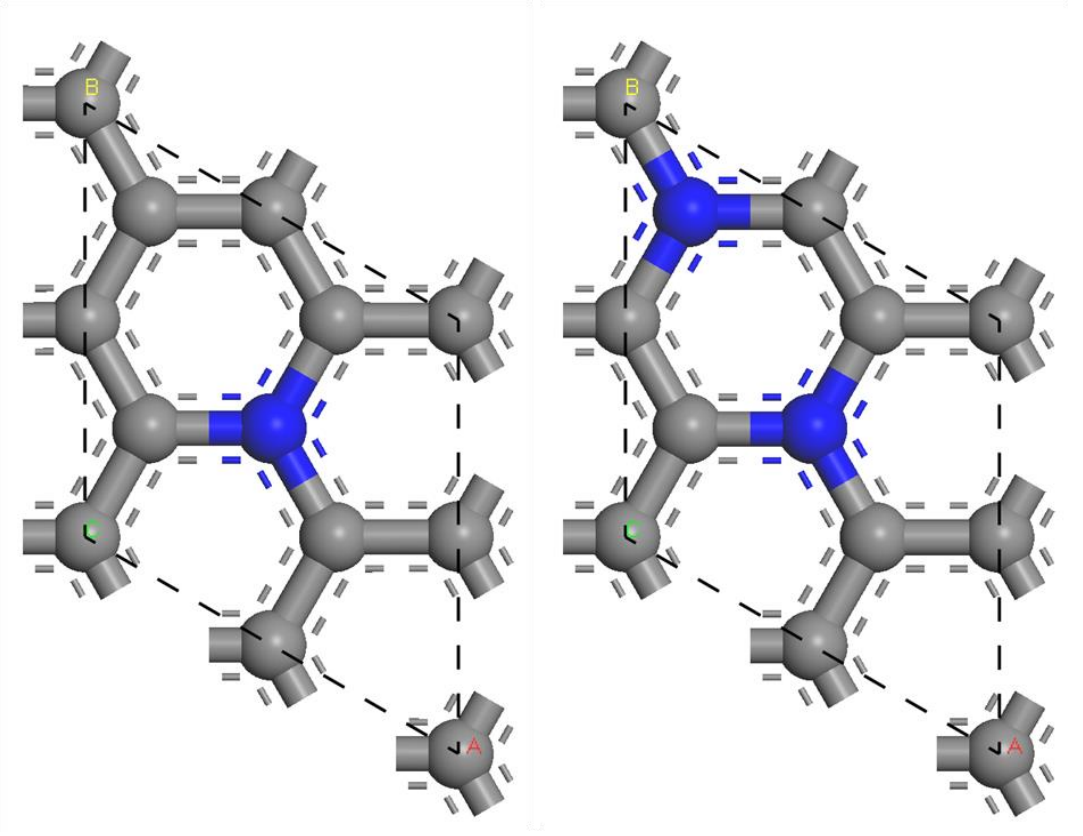




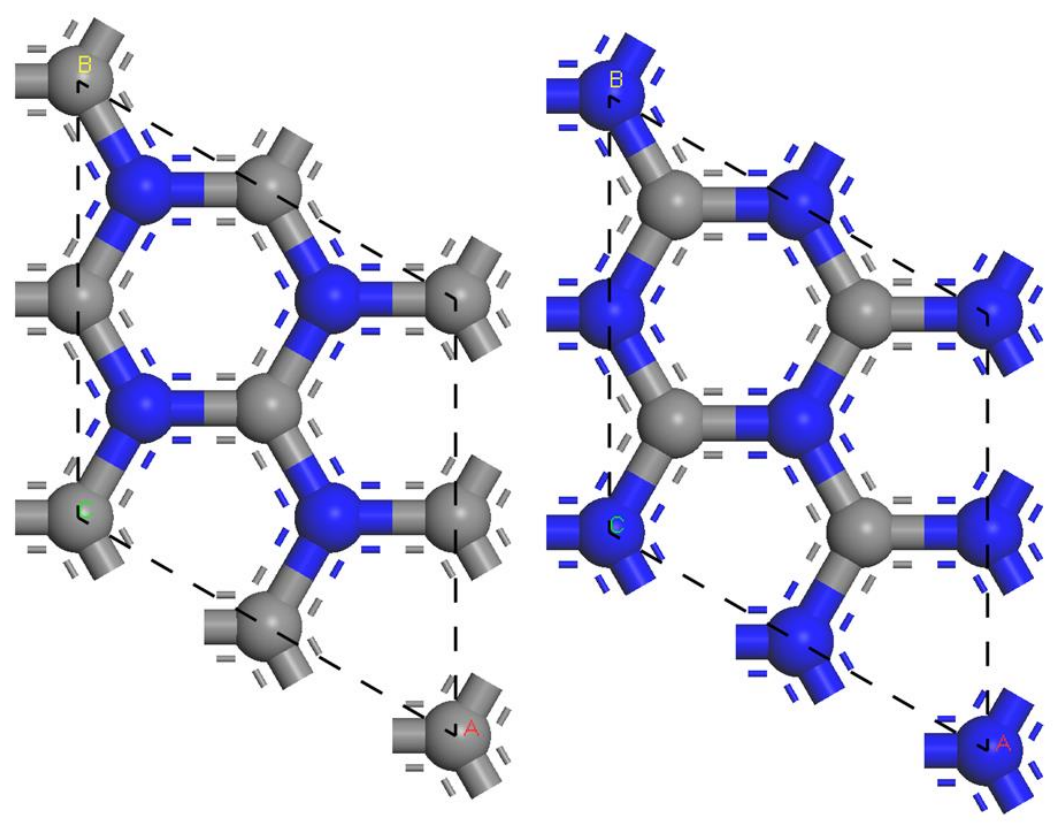

Fig. 4. Optimized structures of 1, 2, 4 and 9 nitrogen atoms doped graphene. The grey and blue balls represent carbon and nitrogen atoms respectively.

Table 2. Total energy, Fermi energy and binding energies of nitrogen doped graphene.

\begin{tabular}{|c|c|c|c|}
\hline Structure & $\begin{array}{c}\text { Total Energy } \\
{[\text { Ha] }}\end{array}$ & $\begin{array}{c}\text { Fermi Energy } \\
{[\mathbf{H a}]}\end{array}$ & $\begin{array}{c}\text { Binding Energy } \\
{[\mathbf{H a}]}\end{array}$ \\
\hline G-1N & 318.8699 & 0.1200 & 2.4807 \\
\hline G-2N & 335.4038 & 0.1264 & 2.3466 \\
\hline G-4N & 368.3771 & 0.0758 & 1.9839 \\
\hline G-9N & 368.3147 & 0.1359 & 1.9215 \\
\hline
\end{tabular}



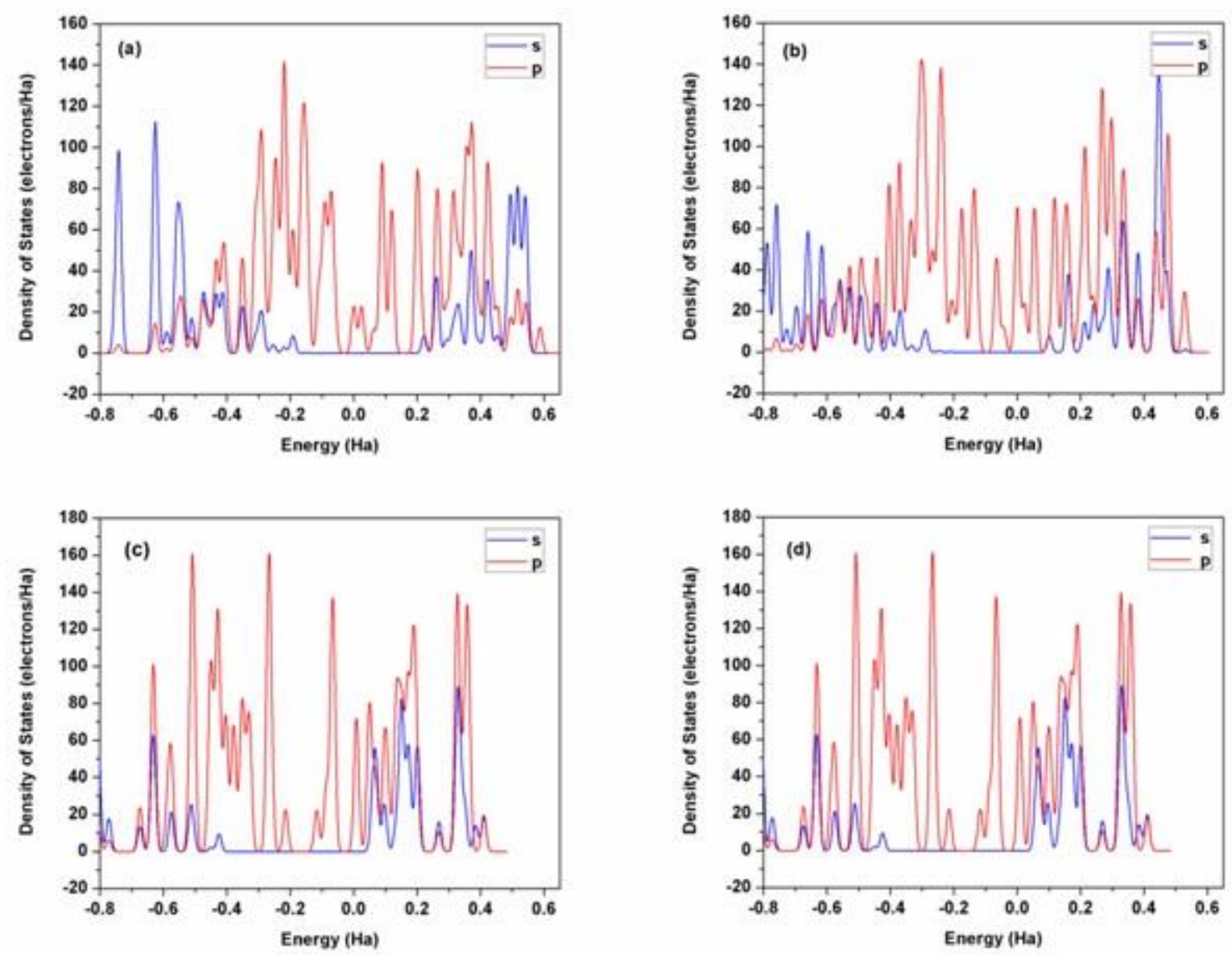

Fig. 5. PDOS of (a) $1 \mathrm{~N}$, (b) $2 \mathrm{~N}$, (c) $4 \mathrm{~N}$ and (d) $9 \mathrm{~N}$ doped graphene. Blue and red curves represent 's' orbital and ' $p$ ' orbital respectively. 

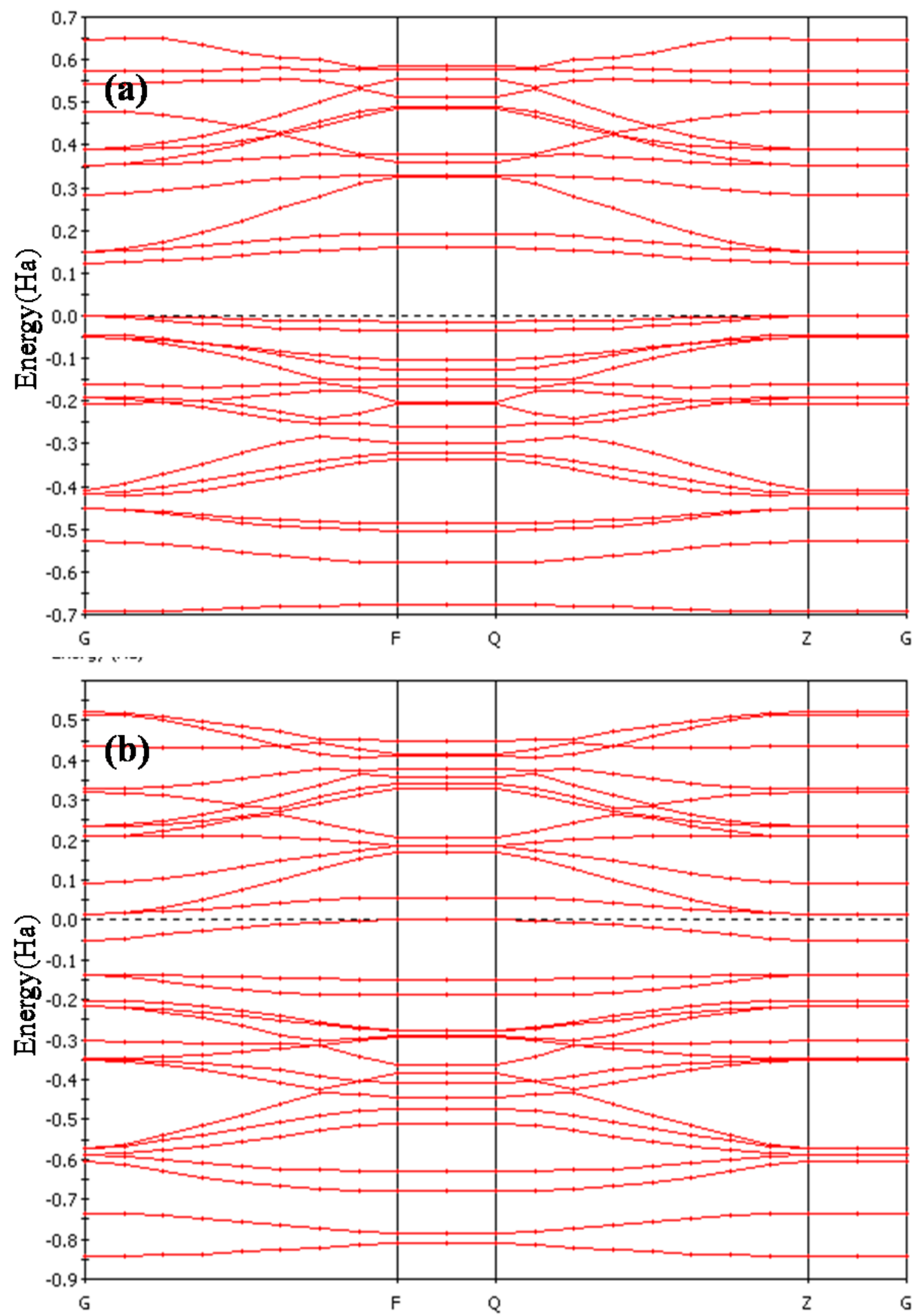

Fig. 6. Band structure of (a) $1 \mathrm{~N}$ and (b) $2 \mathrm{~N}$ doped graphene. 
The density of states of 1, 2,4 and 9 nitrogen atoms doped graphene are shown in Fig. 5 (a-d). Addition of nitrogen dopants drastically changes the DOS near Fermi energy. The band structure also reflects this change (Fig. 6). When 1 nitrogen atom is doped, allowed states observed just above the Fermi energy. In contrast, on doping with 2 nitrogen atoms, energy gap is introduced above Fermi energy. On doping with 4 and 9 nitrogen atoms in graphene, the band gap increases and the system becomes n-type semiconductor.

In order to understand the chemical stability of the doped graphene, we have analysed the Highest Occupied Molecular Orbital (HOMO) and Lowest Unoccupied Molecular Orbital (LUMO). The HOMO-LUMO gap will provide some insight on the chemical stability. The HOMO and LUMO of the pure and doped graphene is shown in Fig. 7 and the HOMO-LUMO gaps are listed in Table 3. The variation of HOMO-LUMO gap in the doped structres is plotted in Fig. 8 and 9 which show drastic changes .

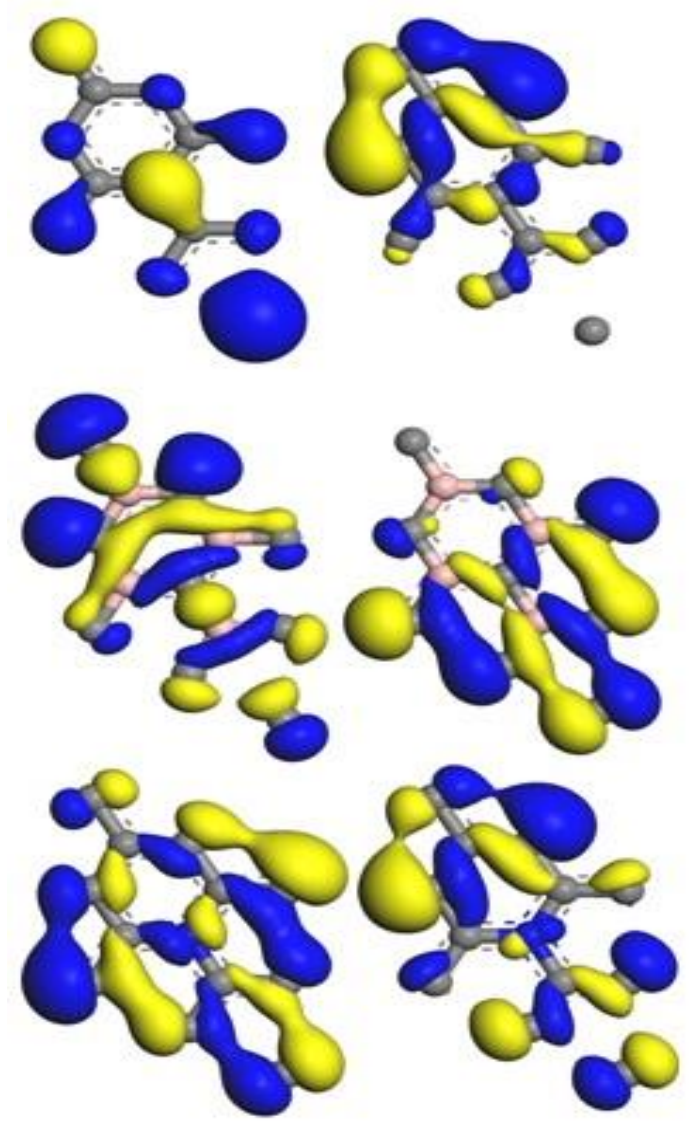

Fig. 7. HOMO (left) and LUMO (right) of pure, 4B and N doped graphene. 
Table 3. HOMO and LUMO energies and HOMO-LUMO gaps of the pure, $\mathrm{B}$ and $\mathrm{N}$ doped graphene.

\begin{tabular}{|c|c|c|c|}
\hline Structure & HOMO [Ha] & LUMO [Ha] & HOMO-LUMO gap [eV] \\
\hline G & 0.2264 & 0.2250 & 0.0381 \\
\hline G-1B & 0.2309 & 0.2188 & 0.3291 \\
\hline G-2B & 0.2280 & 0.2185 & 0.2584 \\
\hline G-4B & 0.2333 & 0.2234 & 0.3291 \\
\hline G-9B & 0.1967 & 0.1846 & 0.0653 \\
\hline B-Sheet & 0.1974 & 0.1950 & 0.2339 \\
\hline G-1N & 0.2386 & 0.2300 & 0.3291 \\
\hline G-2N & 0.2340 & 0.2219 & 0.0898 \\
\hline G-4N & 0.2584 & 0.2551 & 0.4787 \\
\hline G-9N & 0.2427 & 0.2251 & \\
\hline
\end{tabular}

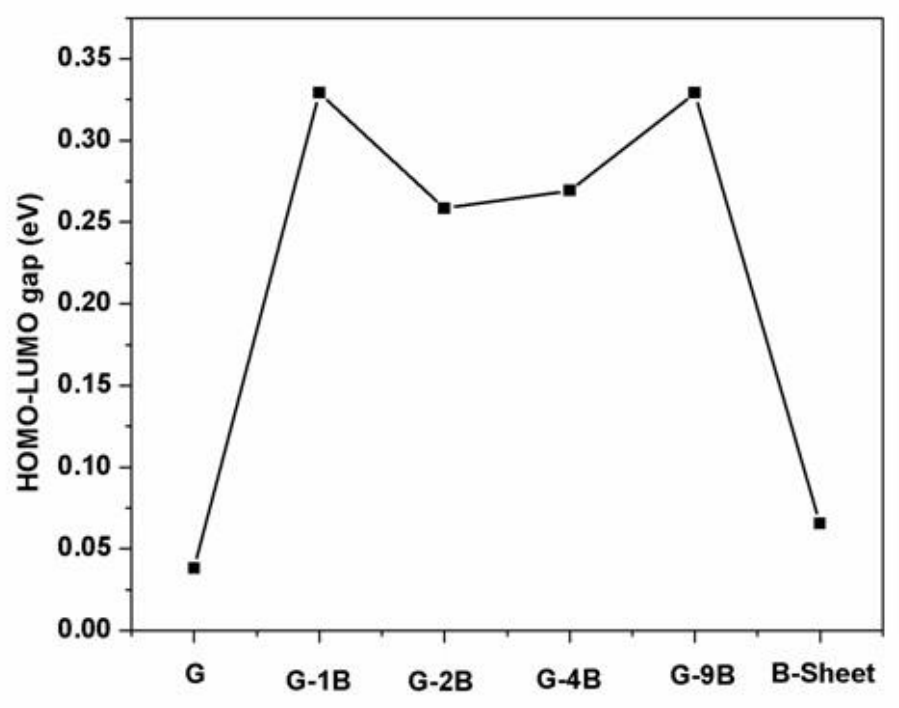

Fig. 8. HOMO-LUMO energy of graphene and boron doped graphene. On increasing the doping concentration, the HOMO-LUMO gap increases and this shows the increased stability. The gap for boron sheet drastically decreases to a low value. 


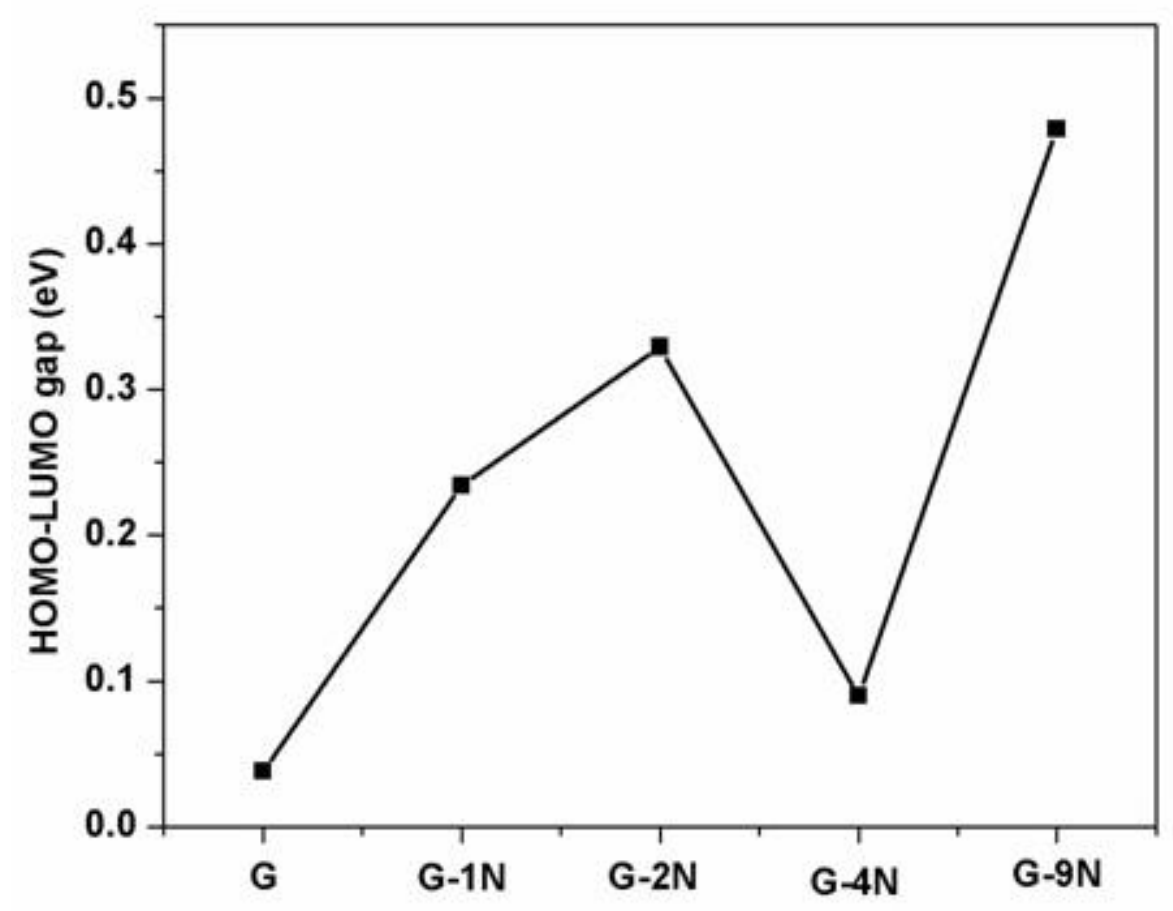

Fig. 9. HOMO-LUMO gap of graphene and N-doped graphene. The $9 \mathrm{~N}$-doped graphene is highly stable as observed.

Based on the HOMO-LUMO gap, it is observed that the doped structures are highly stable than the pure graphene and boron sheets, as seen in Fig. 8 and 9. The graphene, doped with 2B and 4B is less stable compared to $1 \mathrm{~B}$ and 9B-doped graphene with low HOMO-LUMO gap. In the case of nitrogen doped graphene, $9 \mathrm{~N}$-doped system is highly stable with large HOMO-LUMO gap of $0.4787 \mathrm{eV}$. The stability of pure graphene and $4 \mathrm{~N}$-doped graphene is almost similar comparted to other structures.

Mullikan Charge Analysis. In pure graphene, the Mullikan charge analysis shows that there is no charge on the carbon atoms. When one boron atoms is doped, the charges are distributed and the charge on the doped boron atom is positive with a value of $0.069 \mathrm{e}$. Some of the carbon atoms have negative charges which range from -0.030e to -0.051e. When 2 boron atoms are doped the charge on each boron atoms is $0.057 \mathrm{e}$. All the carbon atoms in this structure have a charge of $-0.019 \mathrm{e}$. On increasing the doping concentration to 3 boron atoms per supercell, the charge on the doped boron 
atoms become negative due to the charge transfer from the nearest carbon atoms. Some of the carbon atoms possess positive charge because of this charge transfer. When all the atoms were replaced with boron atoms to get boron sheet, no significant charge distribution is observed as in pure graphene.

On doping the graphene with nitrogen which has one electron in excess compared to carbon, the charge on the doped $\mathrm{N}$-atom is $-0.518 \mathrm{e}$. All the carbon atoms in this case possess positive charge and their values range from $0.026 \mathrm{e}$ to $0.143 \mathrm{e}$. When the doped nitrogen atom is 2 , the charge on nitrogen atom is $-0.540 \mathrm{e}$. Now the charge on the carbon atoms range from $0.092 \mathrm{e}$ to $0.224 \mathrm{e}$. When nitrogen atom is 4 , the charge on all nitrogen atoms is $-0.464 \mathrm{e}$ and the charge on all the carbon atoms is 0.464e. In 9 nitrogen atoms doped graphene also, similar charge distribution is observed.

Hydrogen Adsorption in B and N-doped Graphene. The $\mathrm{H}_{2}$ molecule adsorption in B and $\mathrm{N}$ doped Graphene has been studied. The structures of B-doped graphene is shown in Fig. 10. The pure graphene adsorbs $\mathrm{H}_{2}$ molecule at a distance of $2.518 \AA$ with a binding energy of $0.35 \mathrm{eV}$. The binding energy of adsorption of $\mathrm{H}_{2}$ molecule decreases on doping the graphene with one boron atom. However, the binding energy exceeds when the number of dopant is 2 . The adsorption distance of $\mathrm{H}_{2}$ molecules and binding energies are listed in Table 3. The $\mathrm{H}_{2}$ adsorption in 4 boron doped graphene drastically affects the structure of graphene. The atom above which the $\mathrm{H}_{2}$ is adsorbed is bulged towards the $\mathrm{H}_{2}$ molecule. The corresponding binding energy is $2.74 \mathrm{eV}$. 

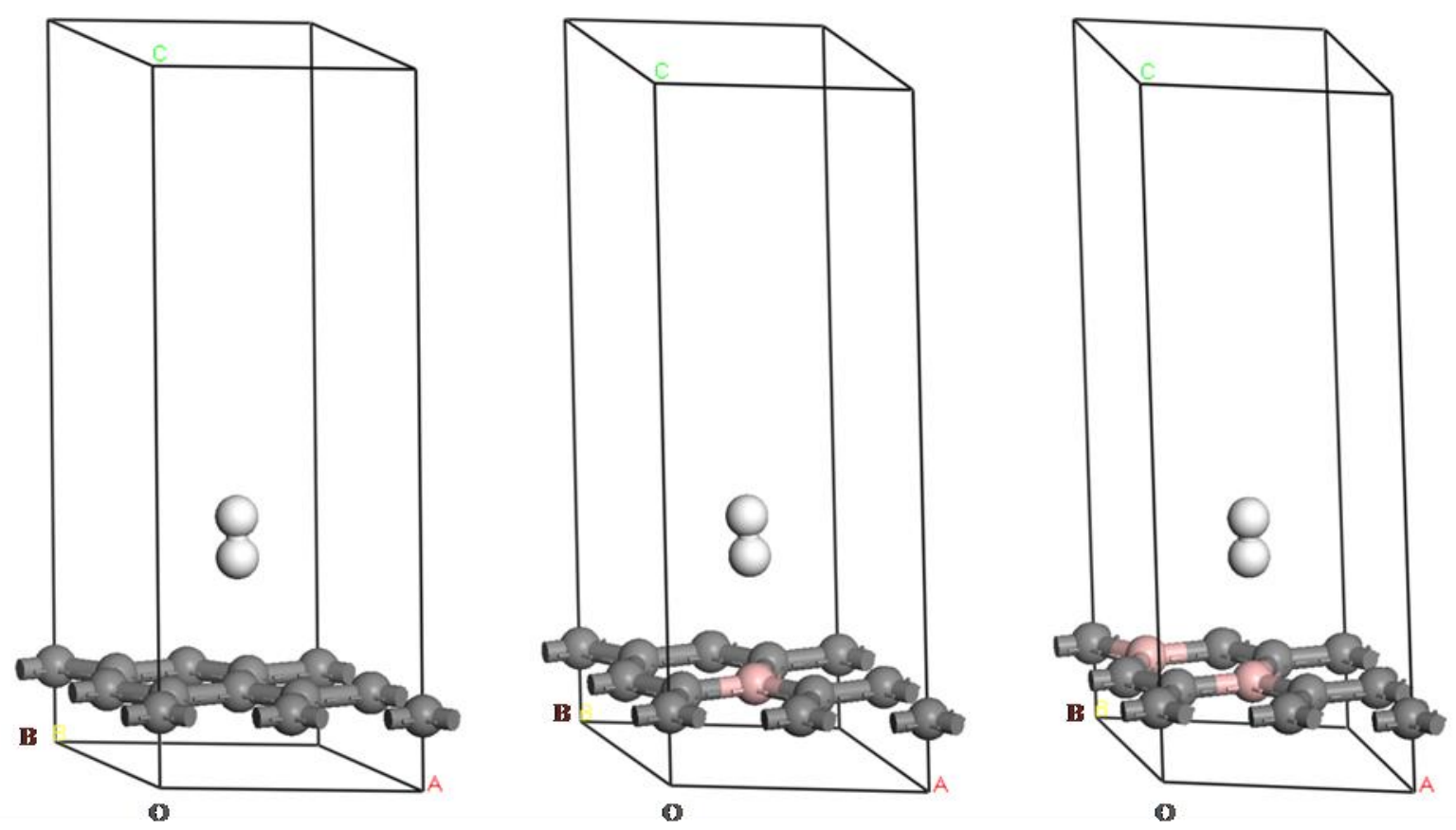

Fig. 10. Optimized structures of pure, 1B and 2B-doped graphene with $\mathrm{H}_{2}$ molecule adsorbed on them. The , 'c' axis was extended to $12 \AA$ to avoid the interference of other layers.

Table 3. $\mathrm{H}_{2}$ adsorption distance and adsorption binding energies of $\mathrm{B}$ and $\mathrm{N}$ doped graphene.

\begin{tabular}{|c|c|c|}
\hline Structures & $\begin{array}{c}\mathbf{H}_{2} \text { Adsorption Distance } \\
{[\AA \mathbf{A}]}\end{array}$ & $\begin{array}{c}\text { Binding Energy of } \mathbf{H}_{2} \\
\text { Adsorption [eV] }\end{array}$ \\
\hline G (Graphehe) & 2.518 & 0.35 \\
\hline G-B & 2.486 & 0.28 \\
\hline G-2B & 2.420 & 2.74 \\
\hline G-4B & 2.208 & 0.21 \\
\hline G-N & 2.417 & 0.33 \\
\hline G-2N & 2.362 & 0.25 \\
\hline G-4N & 2.421 & \\
\hline
\end{tabular}


The $\mathrm{H}_{2}$ adsorption in nitrogen atom doped graphene also has been studied and the adsorption distances listed in Table 3. There is no much change in the binding energy of adsorption and adsorption distance when the number of nitrogen atoms is increased to 2 and 4 .

\section{Conclusion}

The structural changes in the geometry of graphene sheet have been examined using DFT based calculations. Density of states and band structure of graphene have been computed by considering a supercell with periodicity 2 along ' $a$ ' and ' $b$ ' axis. Boron and nitrogen atoms have been doped and corresponding changes in the density of states and band structures have been computed. The addition of boron and nitrogen atoms drastically changes the electronic structure of graphene. HOMO and LUMO are obtained for doped structures. The HOMO-LUMO energy gap widens on increasing the number of boron atoms. In addition to the electronic properties, $\mathrm{H}_{2}$ adsorption has been studied by considering a box with $12 \AA$ along 'c' axis. The binding energy of adsorption on boron and nitrogen doped graphene increases or decreases depending on the number of doping atoms.

\section{Acknowledgment}

One of the authors (MM) would like to thank the Department of Science and Technology (DST), New Delhi, for providing fund for DMol ${ }^{3}$ through Fast Track Young Scientist Scheme.

\section{References}

[1]J. Zhao, R.H. Xi, Electronic and photonic properties of doped carbon nanotubes, J. Nanosci. Nanotechnol. 3 (2003) 459-478.

[2]X.R. Wang et al, N-doping of graphene through electrochemical reactions with ammonia, Science 324 (2009) 768-771.

[3]T.X. Cui et al, Synthesis of nitrogen-doped carbon thin films and their applications in solar cells, Carbon 49 (2011) 5022-5028. 
[4]C. Chen, C. Park, B.W. Boudouris, J. Horng, B. Geng, C. Girit, A. Zettl, M.F. Crommie, R.A. Segalman, S.G. Louie, F. Wang, Controlling inelastic light scattering quantum pathways in grapheme, Nature 471 (2011) 617-620.

[5]L.T. Qui et al, Nitrogen doped graphene as efficient metal-free electrocatalyst for oxygen reduction in fuel cells, ACS Nano 4 (2010) 1321-1326.

[6]Y. Wang et al, Nitrogen doped graphene and its application in electrochemical biosensing, ACS Nano 4 (2010) 1790-1798.

[7]C. Zhou, J. Kong, E. Yenilmez, H. Dai, Modulated Chemical Doping of Individual Carbon Nanotubes, Science 290 (2000) 1552-1555.

[8]P. Ayala, R. Arenal, M. Rummeli, A. Rubio, T. Pichler, The doping of carbon nanotubes with nitrogen and their potential applications, Carbon 48 (2010) 575-586.

[9]R.T. Lv et al, Open ended, N-doped carbon nanotubes-graphene hybrid nanostructures as highperformance catalyst support, Adv. Funct. Mater. 21 (2011) 999-1006.

[10] T.B. Martins, R.H. Miwa, Antonio J.R. da Silva, A. Fazzio, Electronic and transport properties of boron-doped graphene nanoribbons, Phys. Rev. Lett. 98 (2007) 196803-1-4.

[11] E. Cruz-Silva, Z. M. Barnett, B. G. Sumpter, V. Meunier, Structural, magnetic, and transport properties of substitutionally doped graphene nanoribbons from first principles, Phys. Rev. B 83 (2011) 155445-1-9.

[12] H. Lee, J. Li, G. Zhou, W. Duan, G. Kim, J. Ihm, Room-temperature dissociative hydrogen chemisorption on boron-doped fullerenes, Phys. Rev. B 77 (2008) 235101-1-5.

[13] R.H. Miwa, T.B. Martins, A. Fazzio, Hydrogen adsorption on boron doped graphene: an ab initio study, Nanotechnology 19 (2008) 155708-1-7.

[14] L. Firlej, Sz. Roszak, B. Kuchta, P. Pfeifer, C. Wexler, Enhanced hydrogen adsorption in boron substituted carbon nanospaces, J. Chem. Phys. 131 (2009) 164702-1-4.

[15] Y.G. Zhou, X.T. Zu, F. Gao, J.L. Nie, H.Y. Xiao, Adsorption of hydrogen on boron-doped graphene: A first-principles prediction, J. Appl. Phys. 105 (2009) 014309. 
[16] S. Mukherjee, T.P. Kaloni, Electronic properties of boron- and nitrogen-doped graphene: a first principles study, J. Nanopart. Res. 14 (2012) 1059-1-5.

[17] Z.H. Zhu, G.Q. Lu, H. Hatori, New insights into the interaction of hydrogen atoms with boron-substituted carbon, Phys. Chem. B 110 (2006) 1249-1255.

[18] Z. Zhou, X. Gao, J. Yan, D. Song, Doping effects of B and N on hydrogen adsorpti on in single-walled carbon nanotubes through density functional calculations, Carbon 44 (2006) 939947.

[19] S. Yu, W. Zheng, Effect of N/B doping on the electronic and field emission properties for carbon nanotubes, carbon nanocones, and graphene nanoribbons, Nanoscale 2 (2010) 1069-1082.

[20] J. Saloni, W. Kolodziejczyk, S. Roszak, D. Majumdar, G. Hill Jr, J. Leszczynski, Local and global electronic effects in single and double boron-doped carbon nanotubes, J. Phys. Chem. C 114 (2010) 1528-1533.

[21] B. Delley, Analytic energy derivatives in the numerical local-density-functional approach, J. Chem. Phys. 94(11) (1991) 7245-7250.

[22] J. P. Perdew, Y. Wang, Accurate and simple analytic representation of the electron-gas correlation energy, Phys. Rev. B 45(23) (1992) 13244-13249. 